index ocomunicación | no 10(1) 2020 | Páginas 241-259

E-ISSN: 2174-1859 | ISSN: 2444-3239 | Depósito Legal: M-19965-2015

Recibido el 16_02_2020 | Aceptado el 13_09_2020 | Publicado el 15_10_2020

\title{
ESTUDIO DEL COMPORTAMIENTO \\ DE CONSUMO DEL SURFISTA \\ ESPAÑOL. UNA APROXIMACIÓN \\ DESDE LA PERSPECTIVA \\ PROFESIONAL
}

\author{
STUDY OF CONSUMPTION BEHAVIOR \\ OF THE SPANISH SURFER. AN APPROACH
}

FROM A PROFESSIONAL PERSPECTIVE

https://doi.org/10.33732/ixc/10/01Estudi

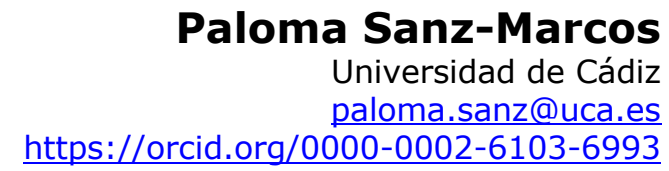

Gloria Jiménez-Marín

Universidad de Sevilla

gloria jimenez@us.es

https://orcid.org/0000-0003-0252-3975 
Resumen: Este artículo realiza un análisis de la práctica y consumo del surf en España con objeto de profundizar en el estudio del surfista español desde una perspectiva de consumo. A través de una metodología de enfoque cualitativo basada en la realización de un panel de expertos que cuenta con los representantes de las ocho marcas más representativas del surf español y la celebración de cuatro focus groups integrados por surfistas españoles, se persigue conocer si tanto la práctica como el consumo de esta actividad conlleva algún tipo de implicación social en su condición como espacio de identificación colectiva. Los resultados indican que, si bien se trata de un deporte plenamente desarrollado socialmente, no existen indicios de que su práctica motive o fortalezca vínculos sociales entre los surfistas españoles.

Palabras clave: surf; consumo; socialización; identidad; marca.

Abstract: This article analyzes the practice and consumption of surfing in Spain in order to broaden the study of the Spanish surfer from a consumption perspective. Through a methodology of qualitative approach based on the realization of a panel of experts with the eight most representative managers of Spanish surfing brands and the celebration of four focus groups integrated by Spanish surfers, it is intended to know if both the practice as the consumption of this activity implies some kind of social implication in its condition as a space for collective identification. The results indicate that, although it is a fully socially developed sport, there is no evidence that its practice motivates or strengthens social ties between Spanish surfers.

Keywords: Surf, Consumption; Socialization; Identity; Brand. 


\section{Introducción}

El surf, en su sentido más general, consiste en el acto de mantenerse en pie encima de una tabla y guiarla a través de la superficie de la cara de una ola rompiente (Booth, 1999). Esta disciplina ha sido investigada por gran variedad de vertientes académicas de entre las que se subrayan la antropología (Rutsky, 1999; Thorne, 1976), la sociología (Irwin, 1973; Ford y Brown, 2006) o la historia (Finney y Houston, 1966). El atractivo multidisciplinar de esta actividad bien puede deberse al origen de su desarrollo, que, acompañado de una serie de condicionantes sociales y culturales, ha supuesto verdaderas implicaciones en el desarrollo de la historia (Thorne, 1976).

Delimitado en muchas ocasiones como deporte, el carácter de esta actividad ha sido ampliamente debatido entre los académicos, que han tratado el tema surgiendo perspectivas que se posicionan favorablemente hacia la consideración del surf como un estilo de vida (Farmer, 1992). Es por ello por lo que algunos autores han descrito esta actividad como un tipo de lifestyle sport (Wheaton, 2004) debido las implicaciones que superan lo estrictamente deportivo (Zavalza, 2020). La vinculación extática de este deporte con la naturaleza (Stranger, 1999) y el carácter espiritual determinado por las implicaciones emocionales surgidas durante su práctica (Anderson, 2013), ofrecen al individuo un sentido vital más allá de lo deportivo (Preston-Whyte, 2002).

Este tipo de experiencias, que han sido igualmente reconocidas en otros deportes en términos de estética (Ford y Brown, 2006), logran crear fuertes relaciones personales entre individuos, lugares y comunidades, desarrollando así una cultura global entre quienes lo practican (Olive, 2016). La literatura académica dedicada a las implicaciones sociales que se desprenden de la práctica y consumo del surf en España es prácticamente inexistente. Es por ello que esta investigación se propone, en primer lugar, realizar un análisis exploratorio de la práctica del surf en España con objeto de ofrecer una primera aproximación a su contexto, presencia y alcance para, a continuación, y de manera más concreta, profundizar en el estudio del surfista español desde una perspectiva de consumo.

Concretamente, se persigue conocer si la práctica y consumo de esta actividad conlleva algún tipo de implicación social en su condición como espacio de identificación colectiva. De este modo, se pretende contribuir a la producción científica en torno a este deporte desde una perspectiva innovadora que abarque el ámbito comunicativo, así como aspectos propios de lo social. 


\section{La práctica del surf en España}

La práctica del surf se encuentra entre uno de los deportes con mayor potencial de proyección económica en España contando cada año con un mayor número de adeptos. La oportunidad de practicar esta actividad en nuestro país España cuenta con casi 8.000 kilómetros de costa-, subraya la importancia de un deporte que, como se puede observar en e1 Gráfico 1, se encuentra en pleno crecimiento y popularidad.

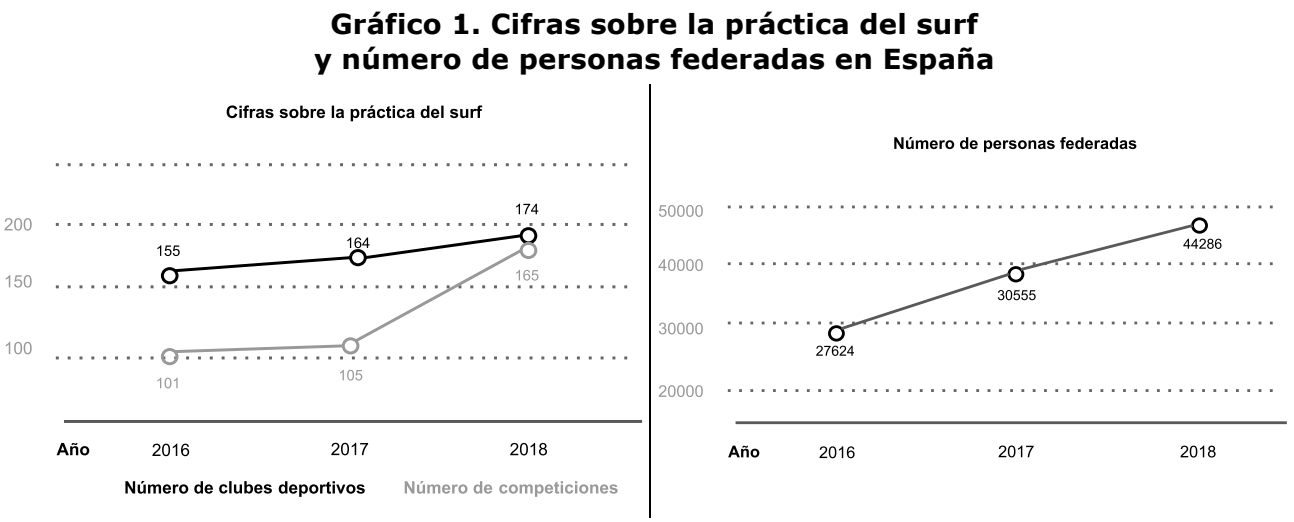

Fuente: elaboración propia a partir de los Anuarios de Estadísticas Deportivas de 2019, 2018 y 2017 elaborados por el Ministerio de Educación Cultura y Deporte.

Este auge es también perceptible desde un punto de vista económico. La industria del surf se encuentra en consolidación gracias a la actividad desarrollada en zonas como el País Vasco (Pellón, 2009), donde se encuentra una importante industria dedicada a la fabricación de tablas de surf, considerándose esta comunidad como la "primera industria "pesada"» de venta de tablas en España» (Esparza, 2016: 91). No obstante, existen otras regiones tradicionalmente vinculadas al surf como son Asturias, Galicia o Cantabria. Esta última reconocida como uno de los núcleos más activos y evolucionados en los primeros años de surf en el territorio español (Esparza, 2013).

Asimismo, es destacable la actividad competitiva y profesional en nuestro país. Existen profesionales de prestigio como Artiz Aramburu, primer español que consigue llegar a clasificarse en el ranking mundial de surf; Jonathan González, canario que ha sido recientemente campeón de Europa en 2016; o jóvenes con potencial como el canario Yael Peña o Iker Amatriain. En cuanto al surf femenino en España, a pesar de contar con un menor número de adeptas ${ }^{1}$,

\footnotetext{
${ }^{1}$ Según datos del Anuario de Estadísticas Deportivas (2016), en España hay alrededor de un 50\% menos de licencias federativas femeninas que masculinas.
} 
existen profesionales como la asturiana Lucía Martiño, Leticia Canales o Garazi Sánchez, entre otras.

Otros datos que reflejan la relevancia del sector es la prolífica generación de contenidos mediáticos en torno a esta actividad. Radios especializadas como es el caso de Hemisferio Surf y otras de carácter general que incluyen la predicción de olas para surfistas, como radio Euskadi con el programa de Rogelio Blasco (Esparza, 2015) así lo demuestran. Además, encontramos revistas como «Surfer Rule, Tres60, Radical Surf, Surf Time, Surf Limit y Surfland [que] son, hoy por hoy, las revistas especializadas que se editan en nuestro país y que gozan de reconocido prestigio internacional» (Pellón, 2009: 11).

El contenido de estas publicaciones suele estar muy relacionado con la práctica especializada de este deporte.,Es por ello que, entre los temas más destacados encontramos reportajes dedicados a destinos de surf, reseñas acerca de nuevos materiales de las diversas temporadas para su práctica, entrevistas a profesionales, secciones de anuncios de compra y venta de material, o agenda de eventos relacionados con este deporte. Es preciso añadir que algunas de ellas han desaparecido o han dejado de editarse en papel, de modo que han surgido nuevas publicaciones - sobre todo en el medio onlineentre las que destacamos Margruesa.

\section{El consumo del surf en España}

Siguiendo a Bouchet, Hillairet y Bodet (2013), el crecimiento del sector deportivo y recreativo está fuertemente impulsado por el desarrollo de las marcas. De hecho, estudios recientes como el de Kakitek (2018) revelan que parte de su espectacular desarrollo ha sido apoyado por su reciente consideración como deporte olímpico. El consumo deportivo a través de las marcas se erige como una oportunidad para manifestar la identidad de los consumidores que incluso puede llegar a afirmar el sentido de pertenencia o la adquisición de roles sociales (Hoye et al., 2015). Stranger (2010) sostiene que entre los surfistas existe una conciencia colectiva que se manifiesta mediante las experiencias de consumo y que se refuerza a través de los momentos de sociabilidad que tienen lugar dentro y fuera del mar.

En efecto, el surf supone una actividad trascendental en la vida de los que lo practican que, a pesar de manifestarse como una experiencia individual, a menudo es compartida con otros surfistas. Se trata de una cultura de compromiso en la que se crean relaciones entre personas, lugares y comunidades, desarrollando así una cultura global entre los que lo practican (Olive, 2016). Beaumont y Brown (2016), quienes hacen un estudio del 
comportamiento de los surfistas de la zona de Cornualles en el sur de Inglaterra, demuestran que en sus playas existe un sentimiento común que se manifiesta en una conciencia compartida entre los surfistas de la zona que entienden el surf como una forma de vida. De este modo, aseguran que surge un fuerte sentido de conexión en torno a la ubicación y a la comunidad de surfistas que los hace compartir un estilo de vida más allá del deporte. No obstante, siguiendo a Del Giudice y Oliveros (2014), esta conexión puede llegar a ser violenta. Los autores afirman que pueden existir jerarquías dentro del agua manifestadas en lo que denominan como el «derecho a ser territoriales» (2014: 311). Si bien se pueden identificar ciertos vínculos con respecto a un lugar, es cierto que la escasez de olas propicia comportamientos agresivos con aquellos surfistas que no son asiduos al lugar defendido y frecuentado por los locales. Este tipo de comportamientos violentos en grupos sociales ha sido identificado por la comunidad científica bajo el nombre de subcultura de consumo: una comunidad cuyos miembros se identifican a partir de un compromiso con una clase de producto de consumo, nombre o actividad en particular, y que poseen un identificable estructura social y jerarquía; un espíritu único, una serie de valores compartido y interés por la marginalidad, el rechazo a lo instaurado y por la experimentación de la diferencia respecto a los demás (Schouten y McAlexander, 1995). A este parecer, las recientes investigaciones de Towner y Lemarié (2020), destacan que, si bien esa reacción violenta supone una forma de limitar e identificar a los miembros de la comunidad, refleja cierto sentido de solidaridad expresado entre los miembros de las comunidades de surfistas.

En particular, el aumento de la relevancia del consumo de deportes y las prácticas de ocio conllevan la comunicación y mantenimiento de la identidad de los individuos debido a la capacidad de expresión cultural que estas actividades son capaces de expresar (Wheaton, 2004). En el caso concreto de las marcas de surf, estas funcionan como generadoras de un estilo específico y una identidad para una determinada comunidad apuntando hacia símbolos de identidad (Bouchet, Hillairet y Bodet, 2013).

No obstante, la diversificación de esta industria contempla desde materiales duros como tablas de surf y trajes de neopreno, hasta ropa y accesorios que atraen a un público que va más allá del mero deportista y que busca un estilo de vida distintivo que comunique una identidad social, particular y exclusiva (Wheaton, 2004). Esta diversidad ha venido generando una situación particular en el consumo por parte de los surfistas. Irwin (1973) advierte que, en torno a 1958, cuando el surf comienza a ganar popularidad, numerosos individuos buscan en la práctica del surf incorporar un estilo de vida considerado como auténtico. Esta oleada de adeptos provoca, según el 
autor, un rechazo por parte de los ya consolidados surfistas que niegan a estos últimos cualquier tipo de legitimidad en su discurso.

De alguna manera, parece que el mercado en torno a este deporte conlleva una diferenciación de varios tipos de públicos. Así lo entiende Donnelly (2006), quien en su trabajo acerca de los deportes extremos destaca que es necesario diferenciar entre varios tipos de consumidores: los reconocidos como «auténticos participantes», y los denominados como «wannabes ${ }^{2}$ » quienes consumen todo tipo de material destinado para la práctica del mismo, haciéndose pasar por deportistas experimentados sin necesidad siquiera de practicar el deporte.

Estos últimos, los considerados como participantes no auténticos parecen encontrar en el consumo de las marcas de surf un modo de comunicar su identidad y mostrar su estatus social. Por su parte, el trabajo de Del Giudice y Oliveros lo expone de manera similar cuando en su estudio sobre el surf en Colombia destacan la presencia de los llamados 'wanna' para referirse a aquellos surfistas que no han aprendido a surfear adecuadamente y no muestran un compromiso con el deporte y consumen bienes «para proyectar a la sociedad que son surfistas» (2014: 309).

Esta premisa se acerca a las teorías sobre el consumo conspicuo elaboradas por el sociólogo Thorstein Veblen por las que se afirma que los individuos consideran el consumo de ciertos bienes como una expresión del estatus social y un modo de demostración de la identidad hacia a los demás que permite identificarse con determinadas comunidades sociales (Veblen, 1989). Esta necesidad de consumo, con objeto de comunicar la identidad a los demás (Yildiz y Yildiz, 2020), es de suma importancia para el sentido de pertenencia de los individuos desde un punto de vista social. La filiación a ciertos grupos es una fuente de identidad para el sujeto, esto es, proporciona similitud entre sus miembros (Turner y Brown, 1978).

A este parecer, Warren y Gibson señalan que las marcas de surf encierran una serie de significados (Hardy, Bennett \& Robards, 2018) cuya legitimidad es incorporada por parte de aquellos que verdaderamente practican este deporte (Warren y Gibson, 2017). De modo que estas marcas dependen de ese conjunto de consumidores core ${ }^{3}$ o nucleares (Donnelly, 2006) para mantener el estatus

\footnotetext{
2 Esta expresión de origen anglosajón se podría traducir literalmente al español como «quiero ser». Se trata de un modo de expresar a alguien que copia o imita aquello a lo que querría parecerse.

${ }^{3}$ La expresión de origen anglosajón core se podría traducir al español como «central» o «núcleo». En este caso hace referencia al núcleo que conforman aquellos consumidores que cuentan con una larga trayectoria en la práctica del surf.
} 
mítico que, a su vez, atrae a un tipo de consumidor de menor implicación con el deporte como pueden ser los wannabes anteriormente mencionados. En definitiva, se desprende que la autenticidad parece cuestionada por diferentes segmentos de practicantes los cuales son comúnmente diferenciados e identificados por sus patrones de consumo (Roberts y Ponting, 2020).

\section{Metodología}

Este trabajo se propone contribuir al conocimiento acumulado sobre el surf en España desde una perspectiva que abarca el ámbito del consumo y las posibles implicaciones sociales que se derivan de su práctica. Si bien existen investigaciones previas que ponen de manifiesto el poder de socialización que otros académicos han reconocido en el consumo y práctica de otros deportes como el fútbol (Llopis-Goig, 2011; Meneses, 2008) o el baloncesto (Merril, Melnick y Chemers, 2013; Walseth, 2006), la literatura académica dedicada al caso del surf en España es prácticamente inexistente. Es por ello que este trabajo parte de las siguientes preguntas de investigación:

- P1. ¿Manifiestan los surfistas españoles algún tipo de vínculo social derivado de la práctica del surf?

- P2. ¿Es el surf un deporte cuyos hábitos de consumo promueven las relaciones sociales?

- P3. ¿Es el surf un deporte socialmente desarrollado en España?

Para dar respuesta a las preguntas de investigación planteadas, se ha llevado a cabo una metodología de enfoque cualitativo que comprende dos técnicas de investigación. En cuanto a la primera de ellas, se ha realizado un panel de expertos conformado por los gestores de las ocho marcas más representativas del surf español. La selección de las marcas escogidas ha considerado los siguientes criterios: marcas de surf originadas en la península ibérica, que cuenten con una red comercial consolidada y que gocen de gran notoriedad entre los surfistas españoles, dando como resultado la participación de las marcas: Pukas, Full\&Cas, Watsay, Soul, Nexo, Feather Fins, Deflow y Vazva.

Es preciso destacar que la búsqueda de las mismas se inició a través de dos canales. Por un lado, se realizó una consulta de la oferta comercial de tiendas online españolas especializadas en surf ${ }^{4}$ para identificar la

\footnotetext{
${ }^{4}$ Las webs consultadas han sido: www.tablassurfshop.com, www.todosurf.com, www.surfmarket.org, www.mundo-surf.com, www.frussurf.com o www.surf3.es. Esta selección responde a los diez primeros puestos de la búsqueda en google de "compra de material surf en España" realizada el 20 de febrero de 2018. Se han considerado las primeras diez posiciones de tiendas de
} 
consolidación de las mismas en el mercado español y, por otro, y para apoyar este criterio, se hizo una consulta a través del localizador virtual de la Oficina Española de Patentes y Marcas bajo la denominación de "Artículos de gimnasia y deporte no comprendidos en otras clases para la práctica de deportes acuáticos. Planchas y tablas para la práctica de surf y nieve, patinetes y monopatines" 5 en su estado de "activo". Esta consulta ha permitido comprobar el origen de las marcas y su vigencia.

Tras el análisis de las marcas seleccionadas, se descartaron para el estudio las siguientes: Slash, dedicada a la fabricación de tablas de surf de origen asturiano, no ha sido incluida en el estudio debido a la imposibilidad de contactar con alguno de sus responsables; las marcas Native Surfboards, de origen malagueño, y la vasca Peta Surfboards, no han participado debido a que, por un lado, gozan de una reducida popularidad local (tan solo fueron identificadas en los grupos andaluz y vasco respectivamente) y, por otro, dada su dedicación a la fabricación de tablas de surf de tipo artesanal, no se han encontrado disponibles en las webs consultadas. Por último, las marcas Hotties, dedicada a la fabricación de neoprenos, y CeCe Surfboards, dedicada a la fabricación de tablas de surf, han sido igualmente descartadas debido a que se encuentran en declive y proceso de cierre inminente.

Atendiendo a Gaitán y Piñuel (1998), la técnica del panel de expertos permite desarrollar una construcción teórica que permite realizar una posterior aproximación a un objeto de estudio. En este caso, y como aconseja Landeta (1999), se ha considerado exponer el proceso de forma anónima coincidiendo con la petición de sus participantes.

El cuestionario estándar preparado y enviado al panel de expertos consistió en las siguientes cinco preguntas:

- PPE.1- ¿Considera que el surf es un deporte que facilite las relaciones sociales?

- PPE.2.- ¿Definiría el surf como un deporte solitario o colectivo?

- PPE.3- Entre los objetivos de las posibles promociones y eventos que organiza su marca, ¿se promueve el encuentro y relación entre los surfistas?

- PPE.4- ¿Cree que su marca es consumida solo por surfistas? ¿Es habitual encontrar entre los consumidores de su marca personas que no practiquen surf?

origen español descartando así las siguientes restantes de procedencia extranjera: www.bluetomato.com, www.magicseaweed.com, www.wetsuitwarehouse.com y www.wetsuitoutlet.co.uk.

${ }^{5}$ Esta denominación es el criterio de búsqueda más afín al material para la práctica de surf disponible en esta oficina para localizar marcas de surf españolas. 
- PPE.5- ¿Cree que su marca podría sobrevivir si únicamente contara con el público objetivo no experto o que sencillamente se siente atraído por la cultura del surf a pesar de no practicarlo?

Con respecto a la segunda técnica de investigación y con objeto de apoyar y comparar las respuestas obtenidas por los expertos, se han llevado a cabo cuatro focus group. Esta técnica permite conocer los testimonios y opiniones de los surfistas en tanto como a consumidores y practicantes de este deporte. Estas entrevistas, que responden a un muestreo no probabilístico por conveniencia de 29 participantes, se celebraron en aquellas zonas de la península ibérica donde la práctica del surf tiene mayor presencia, esto es, la zona norte occidental de España en La Coruña (ocho participantes), la zona norte central del cantábrico en Asturias (seis participantes), la zona norte más oriental del cantábrico en el País Vasco (siete participantes) y la zona sur con un grupo celebrado en Andalucía (ocho participantes).

Esta técnica se considera adecuada para estudiar los surfistas debido a que, como indican Taylor, Bogdan y Devault (2015), permite plantear y debatir las posibles apreciaciones y opiniones de un grupo de individuos que comparte algún tipo de vínculo. Asimismo, su eficacia reside en la oportunidad para recoger datos espontáneos por parte de los entrevistados (Walle, 2015) lo cual aporta una amplia perspectiva de las opiniones y experiencia de los surfistas. Esta consideración daría respuesta al objetivo de conocer cómo es la práctica del surf en España al mismo tiempo que permite conocer cómo es la relación desarrollada entre los surfistas.

En cuanto a las preguntas planteadas durante las entrevistas, se consideraron las siguientes ocho preguntas:

- PFG.1- ¿Es el surf un deporte solitario?

- PFG.2- ¿Se puede practicar surf en solitario?

- PFG.3- ¿En qué medida es importante practicar surf rodeado de otras personas?

- PFG.4- Si pudieras elegir, ¿con cuántas personas practicarías surf?

- PFG.5- ¿Diríais que gracias al surf habéis logrado hacer amigos?

- PFG.6- ¿Qué importancia le dais al consumo de marcas de surf?

- PFG.7- ¿Existe gente que consuma marcas de surf y que no practique surf? ¿Qué os parece que ocurra esto?

- P.8- Si vais a un evento relacionado con el surf, ¿es importante para vosotros el evento en sí o la relación que se establece con los demás? 
Tabla 1. Relación entre preguntas de investigación y las preguntas planteadas en el panel de expertos y los focus groups

\begin{tabular}{|l|l|l|}
\hline $\begin{array}{c}\text { Preguntas de } \\
\text { investigación }\end{array}$ & $\begin{array}{c}\text { Preguntas planteadas } \\
\text { en el Panel de Expertos }\end{array}$ & $\begin{array}{c}\text { Preguntas planteadas } \\
\text { en los Focus Groups }\end{array}$ \\
\hline P.1 & PPE1, PPE2 & PFG1, PFG2, PFG3, PFG4, PFG5 \\
\hline P.2 & PPE1, PPE2, PPE3 & PFG8 \\
\hline P.3 & PPE4, PPE5 & PFG6, PFG7 \\
\hline
\end{tabular}

Fuente: elaboración propia.

En definitiva, con el empleo de ambas técnicas se propone dar respuesta a los objetivos planteados desde una perspectiva comparativa que pretende contrastar la información atendiendo tanto al consumidor y practicante del surf, como a los responsables de las estrategias dedicadas al consumo ofreciendo de este modo una visión global del mercado del surf en España.

\section{Discusión de los resultados}

Los resultados obtenidos tras la realización del panel de expertos, así como de los focus groups, manifiestan que el diseño de las entrevistas fue adecuado para la obtención de resultados interesantes para la investigación. Los participantes no presentaron apenas indicios de confusión o problema a la hora de entender las preguntas planteadas y demostraron un amplio conocimiento sobre el deporte.

Concretamente, en el caso de los expertos, se advierte un alto grado de conocimiento en torno al mercado del surf. Los individuos aportaron durante la realización de las entrevistas datos espontáneos acerca de la idiosincrasia de este deporte revelando en la mayoría de los casos un destacado auge económico del mercado. Así lo indica el experto de la marca número 1 cuando anuncia que: "[...] ha habido un cambio abismal en cuanto a presupuestos [...] hace apenas cinco años hemos visto como ahora podemos contar con un mayor presupuesto".

De igual modo, en el caso de los surfistas, la mayoría de los entrevistados asegura que el surf parece un deporte en el que, “[...] aunque la gente aún nos mire raro cuando llevamos tablas [de surf], es increíble como en el agua cada vez hay más gente" (surfista número 23), indicando de este modo que, a pesar de ser todavía un deporte minoritario, se encuentra en pleno desarrollo. Estas respuestas se vinculan directamente con la tercera pregunta de investigación que se cuestiona si, en efecto, el surf es un deporte desarrollado socialmente en España. A tenor de lo planteado, tanto los expertos como los surfistas - aunque en mayor medida estos últimos-, revelan un interesante relato acerca de lo que pudiera parecer un deporte poco accesible para las masas. 
Los expertos comentan que, si bien el surf es un deporte que atrae a un público masivo que no surfea, las estrategias de comunicación llevadas a cabo por parte de las marcas y que se dedican a este tipo de público: "[...] suelen sentar mal a los surfistas de a pie" (experto marca número 3). Esta afirmación apoya las opiniones de los consumidores, como la del surfista número 17, el cual revela que: "Hay ahora una moda del surf que da vergüenza. Esta gente que aparece en la playa sin haber surfeado en la vida pero que va perfectamente equipado". 0 la del surfista número 29 para quien, bajo su punto de vista, es posible hacer una diferencia entre lo que denomina surfero y surfista: "[...] el surfista que es el deportista, el que quiere ir a la playa para hacer surf, ese es el surfista, y el surfero es el que [...] voy a la playa para hacerme fotos".

Estas afirmaciones sugieren cierta correspondencia con las teorías de Veblen (1989) en cuanto al consumo como expresión del estatus social. Parece que entre los distintos grupos de consumidores existe un conjunto que consume estas marcas con objeto de representar cierta identidad que, paradójicamente, no parece ser aceptada por el núcleo de surfistas. Dado que la oportunidad de establecer lazos entre iguales presenta cierta complejidad animada por esa diferenciación en torno a la legitimidad de sus practicantes, el surf no parece ser un escenario de identificación colectiva (Turner y Brown, 1978) o, al menos, entre cierto tipo de individuos.

Esta diferenciación entre los practicantes de surf es recogida, igualmente, por el experto de la marca número 2 cuando sostiene que "[...] es una discusión muy complicada que siempre la tenemos a nivel estratégico porque, claro, te estás limitando a un público, si te amplias, te amplias a otros, pero pierdes tu identidad", sugiriendo, de este modo, el reconocimiento de distintos tipos de públicos basado en la autenticidad de sus prácticas de consumo (Donnelly, 2006). Idea que apoya el experto de la marca número 6 cuando indica que "[...] ahora que hemos ampliado los canales de venta a establecimientos como el Corte Inglés, oyes por ahí que nuestra marca se ha vendido", coincidiendo así con la perspectiva anteriormente comentada de Warren y Gibson (2017) acerca de la pérdida de legitimidad de las marcas debido a su presencia en canales de consumo masivos.

De hecho, algunos participantes, ante las cuestiones relativas al consumo de las marcas de surf, manifiestan cierto desencanto. Es el caso del surfista número 13 quien añade que: "el público se ha cansado de pagar cuatrocientos euros por un neopreno que no está justificado". En esta línea, el surfista número 20 atribuye este desencanto precisamente a esta apertura de las marcas hacia otros mercados no expertos: "[...] no quiero llevar lo que lleva el mainstream", 
manteniendo, así, que el consumo de marcas de surf puede llegar a ser algo incluso ilegítimo.

Para responder a la primera pregunta de investigación — dirigida a conocer si los surfistas manifiestan algún tipo de vínculo social一, los expertos fueron preguntados acerca de si el surf facilitaba las relaciones sociales y si, en cualquier caso, se trataba de un deporte de práctica solitaria. Las respuestas obtenidas se han encaminado de manera unánime hacia la negación de cualquier incitación hacia lo social. Así lo confirma la respuesta del experto de la marca número 4 cuando señala que: "El surf siempre ha sido de pocos y amigos" o el experto de la marca número 8 , quién después de comentar que llevaba muchos años practicando surf, asegura que: "Cuantos menos seamos en el agua, mejor. Por lo que creo que no facilita mucho la creación de nuevos amigos".

Esta tendencia, es igualmente apoyada por los surfistas. Ante la pregunta de si el surf se podría considerar como un deporte solitario, todos los participantes manifiestan que se trata de un deporte completamente individualizado. Las afirmaciones del surfista número 1 así lo reflejan cuando determina que: "[...] yo creo que es un deporte individual. No necesitas estar con más gente". De hecho, el surfista número 12 puntualiza que: "[...] si llega un punto que hay más gente de la cuenta, ya te agobias".

No obstante, se aprecian posturas intermedias en cuanto a la necesidad de practicarlo en compañía, ya que, como afirma el surfista número 4: "Solo no me siento cómodo en el agua. Más que cómodo seguro", justificando así que la necesidad de la presencia de otros puede ser positiva en estrictos términos de seguridad.

Estas apreciaciones apoyan las respuestas obtenidas en torno a la segunda pregunta de investigación que plantea si, en efecto, el surf es capaz de promover las relaciones sociales entre sus practicantes. Los expertos coinciden en que, si bien se organizan numerosos eventos en los que se promueve la participación entre los surfistas, no es común encontrar una destacada asistencia a dichos eventos. Como sostiene el experto de la marca número 2: "Los eventos son prácticamente un modo de apoyar la publicidad, y eso no atrae a los surfistas", reforzando, en un comentario posterior, la idea de que los eventos suelen atraer a ese denominado público surfista del que como se ha descrito anteriormente, parece cuestionada su autenticidad.

Por parte de los consumidores, sus declaraciones vienen a confirmar lo sugerido por los expertos mediante apreciaciones como las de la surfista número 12 quien sostiene sobre los eventos que: "no me «molan» nada. Me siento como que estoy en un sitio en el que no estoy cómoda", demostrando que los eventos no suelen despertar el interés de los surfistas, salvo en 
determinadas ocasiones como las que señala el surfista número 20, quien señala que: "yo es que, o trabajo en el evento o no voy. Normalmente no voy, a no ser que trabaje o colaboremos".

La seguridad en lo rotundo de las respuestas, tanto de los expertos como de los surfistas, contrasta con las investigaciones que otros autores han llevado a cabo sobre el poder de socialización que se deriva de la práctica de otros deportes (Llopis-Goig, 2011; Merril, Melnick y Chemers, 2013) donde parece que las relaciones sociales son un activo complementario a la mera práctica deportiva.

\section{Conclusiones}

A través de este estudio, se ha analizado la práctica y consumo del surf en el territorio español. Al respecto, frente a la falta de indicios en la literatura académica de un análisis de la perspectiva social de los surfistas, pero en consonancia con propuestas aplicadas a otros deportes como las de Llopis-Goig, 2011; Meneses, 2008; Merril, Melnick y Chemers, 2013; y Walseth, 2006, se planteó conocer si, en efecto, la práctica y consumo de esta actividad conllevaba algún tipo de implicación social en su condición como espacio de identificación colectiva.

Sin embargo, los resultados revelan que el surf no puede ser considerado como un deporte facilitador de las relaciones sociales. De hecho, ni siquiera contempla de manera generalizada la posibilidad de practicarlo a través de pequeños grupos o núcleos sociales atendiendo al deseo, ampliamente evidenciado por parte de sus adeptos, de ser practicado en soledad.

Es por ello que el factor individualista parece preponderante sociológicamente: la mayoría de los participantes sostiene que una de las condiciones ideales para la práctica del surf es la posibilidad de surfear en solitario o acompañado del menor número de surfistas posible. Esta cuestión ha sido manifestada reiteradamente a través de apreciaciones que destacan la consideración del surf como un deporte solitario por parte de los entrevistados. De hecho, las motivaciones positivas evidenciadas para compartir este deporte responden a una cuestión de conveniencia por motivos de seguridad ante posibles situaciones de peligro.

De este modo, se observa que la oportunidad para establecer vínculos de tipo social o socializar en el momento de la práctica del deporte o construir un sentido de pertenencia grupal son cuestiones no valoradas, cuando no inexistentes, entre los participantes, quienes manifiestan una preferencia hacia la práctica solitaria de este deporte. De hecho, destaca cierta conexión que se aleja del vínculo amistoso reconociendo cierta violencia entre sus practicantes 
a la hora de defender los lugares de la práctica. Si bien existe una defensa de la territorialidad y una relación jerárquica entre los surfistas, las actitudes de tipo agresivo subrayan ese carácter poco social que encierra la actividad. Por tanto, no se halla un beneficio de conexión social que motive a los surfistas españoles a la práctica de este deporte.

Este hecho supone un claro avance en el conocimiento de un deporte que, si bien se ha manifestado como plenamente desarrollado en nuestro país a tenor de las respuestas obtenidas por los expertos y surfistas que revelaban un claro conocimiento de la práctica, naturaleza y mercado del surf, presenta una modelo de consumo particular condicionado por el nivel de destreza de sus practicantes.

Al contrario de lo que ocurre en otros deportes como el fútbol, en el que la participación y consumo masivos beneficia a la articulación de su mercado, el surf parece excluir precisamente a aquellos practicantes que no responden a ciertos estándares de competitividad. Por parte de los surfistas, estos manifiestan que aquellos individuos que sencillamente visten con marcas de surf o practican de manera irregular la actividad, representan un conjunto de sujetos que no pueden considerarse como auténticos surfistas, llegando incluso a rechazar cualquier tipo de vínculo entre los mismos. Algo que, al mismo tiempo, ocurre entre los propios surfistas experimentados, los cuales revelan que las mejores condiciones para la realización de esta actividad implican una práctica solitaria.

Del mismo modo ocurre en el caso de los expertos, quienes evidencian que la gestión de sus marcas puede hacerse verdaderamente compleja a la hora de desarrollar sus estrategias en tanto deben distinguir correctamente a quién dirigir sus comunicaciones para no perder autenticidad y legitimidad en su imagen de marca. Como ya ocurriera en estudios anteriores, la autenticidad supone un punto de inflexión entre los practicantes que, a través de las prácticas de consumo, llevan a cabo procesos de marginalización entre sus adeptos.

Esta revelación tiene importantes consecuencias en lo relativo a lo estrictamente social pero también en el escenario de lo económico. Dado que no existe una complejidad enfocada a la interacción social alrededor de este deporte, la gestión de este mercado puede verse fuertemente afectada en tanto que no se muestra una vinculación en el impacto cultural de este deporte con respecto al consumidor. A nivel estratégico, la gestión de este deporte supone un gran reto para sus responsables que deben atender a las demandas de un público exigente.

En definitiva, el presente trabajo, si bien no rechaza la posibilidad de que el deporte sea un importante agente socializador en ciertos contextos 
concretos, y para cierto tipo de actividades, sí permite señalar que en el caso del surf no es factible. Como futuras recomendaciones se aconseja testar estas teorías en otros países para aportar una perspectiva más global e integradora. Esta recomendación responde a una de las limitaciones que destacamos en esta investigación. El hecho de analizar el mercado español supone un condicionante restrictivo que puede limitar la aplicabilidad de este estudio. No obstante, este condicionante plantea a su vez posibles futuras líneas de investigación conforme a la aplicabilidad de estas teorías a otros mercados extranjeros. Al mismo tiempo, la combinación de otros enfoques cualitativos como la etnografía podrían resultar interesantes para conocer en mayor detalle las relaciones entre los surfistas, así como sus hábitos de consumo en contextos reales y con mayor precisión.

\section{Referencias bibliográficas}

ANDERSON, J. (2013). Cathedrals of the surf zone: regulating access to a space of spirituality. Social \& Cultural Geography, 15(8), 954-972.

\section{https://doi.org/10.1080/14649365.2013.845903}

BEAUMONT, E. y BROWN, D. (2016). «It's not something I'm proud of but it's... just how I feel»: local surfer perspectives of localism. Leisure Studies, 35(3), 278-295. https://doi.org/10.1080/02614367.2014.962586

Bоотн, D. (1999). Surfing: The cultural and technological determinants of a dance. Culture, Sport, Society, 2(1), 36-55.

Bouchet, P., HILlaireT., D. y BoDET, G. (2013). Sport Brands. New York: Routledge.

DEL GiUdicE, O. N. y Oliveros, M. G. (2014). Análisis descriptivo de la dinámica del surf como práctica social. Telos, 16(2), 300-314.

Donnelly, M. (2006). Studying Extreme Sports. Beyond the Core Participants. Journal of Sport \& Social Issues, 30(2), 219-224.

ESPARZA, D. (2013). La historia del surf en España: de las primeras expediciones al Pacífico a los años 70. Olomouc: Univerzita Palackého v Olomouci.

ESPARZA, D. (2015). Hacia una historia del surf en Andalucía: Génesis y consolidación del surf en Cádiz y Málaga. Materiales para la Historia del Deporte, 13, 47-62.

ESPARZA, D. (2016). Historia del surf en España. De Magallanes a los años 80. Edición Olo Surf History.

FARMER, R. (1992). Waves of semiosis: Surfing's iconic progression. Journal of Sport Behavior, 15(3), pp. 241-257.

FinNEY, B. y Houston, J. (1966). Surfing: A History of the Ancient Hawaiian Sport. San Francisco: Pomegranate Artbooks. 
FORD, N. y BRown, D. (2006). Surfing and social theory: Experience, embodiment and narrative of the dream glide. London: Routledge.

GAITÁN, J. A y PIÑUEL, J. L. (1998). Técnicas de investigación en comunicación social. Elaboración y registro de datos. Madrid: Síntesis.

HARDY, A.; BENNETT, A. y RoBARDS, B. J. (2018). Neo-Tribes: Consumption, leisure and tourism. London: Palgrave Macmillan.

HoYE, et al. (2015). Sport management. Principles and applications. London: Routledge.

IRWIN, J. (1973). Surfing: The natural history of an urban scene. Urban life and culture, 2(2), 131-160.

KAKITEK, A. (2018). Application of Aaker's brand personality scale on human brands in surf sports. Journal of Management and Business Administration, 26(4), 11-31.

LANDETA, J. (1999). El método Delphi. Una técnica de previsión para la incertidumbre. Barcelona: Ariel.

LLOPIS-GOIG, R. (2011). Práctica, consumo e identificación. La primacía del fútbol en las pautas de ocio de la sociedad española. Panorama Social, (14), 136-148.

MELNICK, M. J., y CHEMERS, M. M. (1974). Effects of group social structure on the success of basketball teams. Research Quarterly. American Alliance for Health, Physical Education and Recreation, 45(1), 1-8.

MENESES, J. (2008). El fútbol nos une: socialización, ritual e identidad en torno al fútbol. Culturales, 4(8), 101-140.

MorGan, D. L. (1998). The Focus Group Guidebook. Thousand Oaks: Sage.

OLIVE, R. (2016). Going surfing/doing research: Learning how to negotiate cultural politics from women who surf. Continuum, 30(2), 171-182.

PELlón, J. (2009). Guía del surf en España. Técnicas, playas surfeables, información turística y práctica y los mejores destinos internacionales. León: Destino Everest.

PRESTON-Whyte, R. (2002). Constructions of Surfing Space at Durban, South Africa. Tourism Geographies: An International Journal of Tourism Space, Place and Environment, 4(3), 307-328.

https://doi.org/10.1080/14616680210147445

ROBERTS, M. y PONTING, J. (2020) Waves of simulation. Arguing authenticity in an era of surfing the hyperreal. International Review for the Sociology of Sport, 55(22), 229-245.

RUTSKY, (1999). Surfing the Other: Ideology on the Beach. Film Quarterly, 52(4), 12-23.

SCHOUTEN, J. W. y MCALEXANDER, J. H. (1995). Subcultures of consumption: An ethnography of the new bikers. Journal of consumer research, 22(1), 43-61. 
STRANGER, M. (1999). The aesthetics of risk: A study of surfing. International review for the Sociology of Sport, 34(3), 265-276.

STRANGER, M. (2010). Surface and substructure: beneath surfing \& commodified surface. Sport in Society, 13(7-8), 1117-1134. https: / / doi.org/10.1080/17430431003780054

TAYLOR, S., BOGDAN, R. y DEVAULT, M. (2015). Introduction to qualitative research methods: A guidebook and resource. New Jersey: John Wiley \& Sons.

ThORNE, T. (1976). Legends of the Surfer Subculture: Part One. Western Folklore, 35(3), 209-217.

TOWNER, N. y LEMARIÉ, J. (2020). Localism at New Zealand surfing destinations: Durkheim and the social structure of communities. Journal of Sport \& Tourism, Online first, 1-20.

TURNER, J. y BROWN R. (1978). Social status, cognitive alternatives and intergroup relations. En TAJFEL, H. Differentiation between social groups: Studies in the social psychology of intergroup relations (pp. 201-234). London: Academic Press.

VEBLEN, T. (1994): The Theory of the Leisure Class. London: Oxford University Press.

WALLE, A. (2015). Quialitative reseach in business: A practical Overview. New Castle: Cambridge Scholars Publishing.

WALSETH, K. (2006). Sport and belonging. International review for the sociology of sport, 41(3-4), 447-464.

WARREN, A. y GIBSON, C. (2017). Subcultural enterprises, brand value, and limits to financialized growth: The rise and fall of corporate surfing brands. Geoforum, 86, 177187.

https: / / doi.org/10.1016/j.geoforum.2017.09.017

WhEATON, B. (2004). Understanding Lifestyle Sport: Consumption, Identity and Difference. London: Routledge.

YILDIZ Y. y YILDIZ K. (2020). Brand Equity on Surf Destinations: Alaçati Sample. En RATTEN V. (eds). Sport Entrepreneurship and Public Policy. Contributions to Management Science, (pp. 91-102). Berlin: Springer.

ZAVALZA, D. (2020). Bob McKnight and Quiksilver: surf shorts, stock exchanges, and bankruptcy. Sport in Society, Cultures, Commerce, Media, Politics, Online first, 1-13. New York: Routledge. 